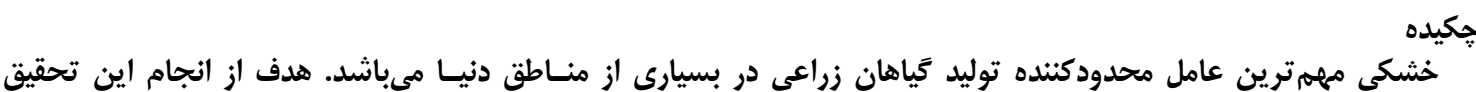

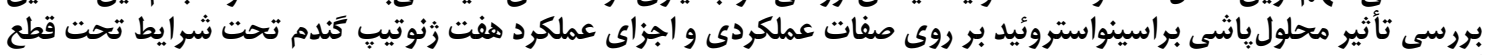

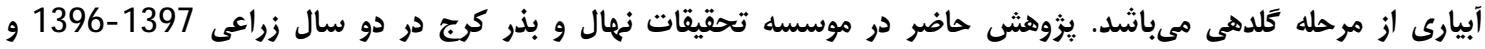

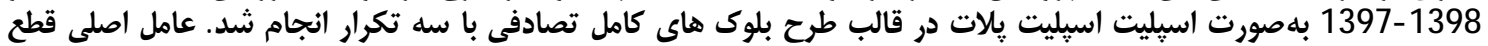

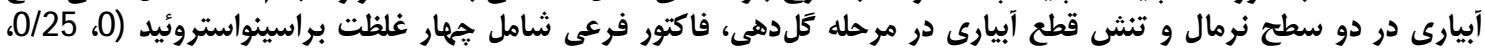

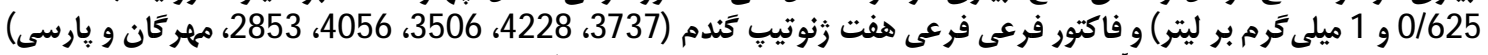

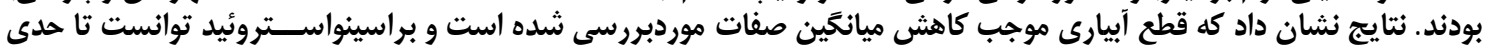

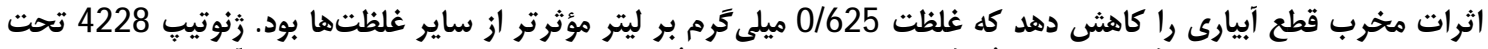

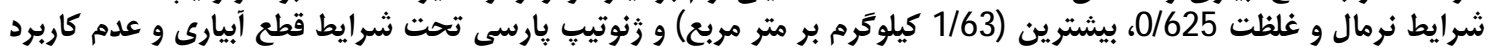

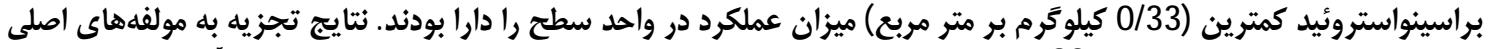

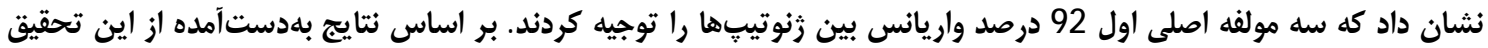

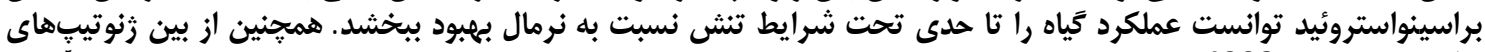

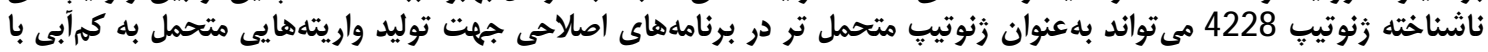
عملكرد مناسب قرار تيرد. وازههاى كليدى: محلولياشى، كلدههى، عملكرد بيولوزيك، تَندم

تأثير قرار مىدهد و با تأثير بر فرايندهاى آنزيمى كه بلطور

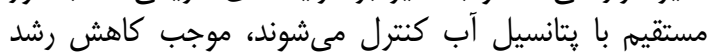

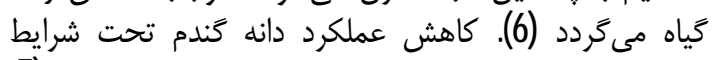

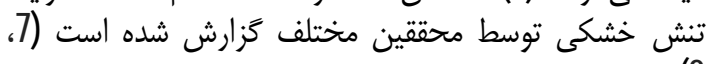

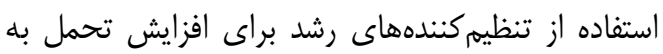

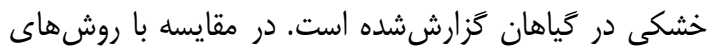

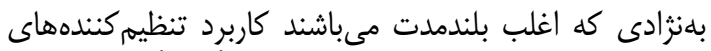
رشد ازجمله براسينواستروئيد آسانتر است (109)

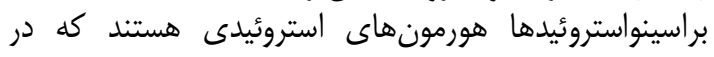

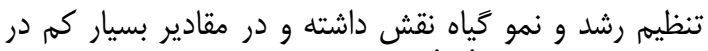

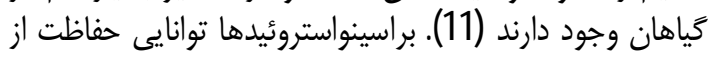

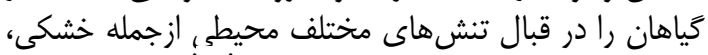

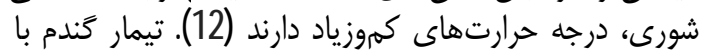

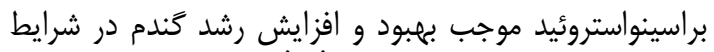

تنش شورى و خشكى شده است (13).

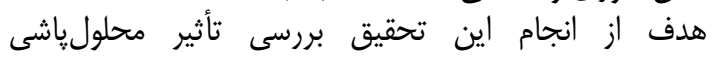

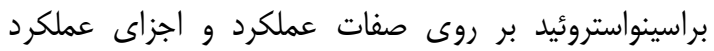
هفت زنوتيب كَندم تحت شرايط قطع آبيارى در مرحله كلدهى مى باشد.
مقدمه

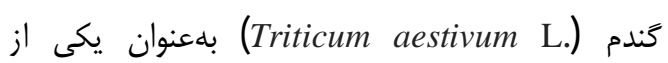

مهرمترين محصولات زراعى ايران و جهان از لحاظظ عملكرد، إنان

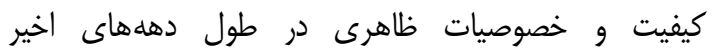

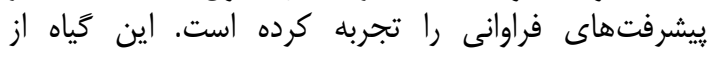

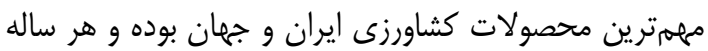

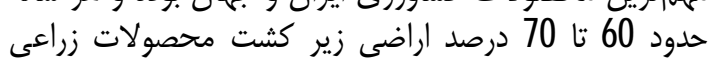

كشور را به خود اختصاص مى دهى دهد (1).

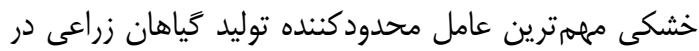

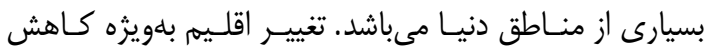

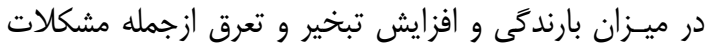

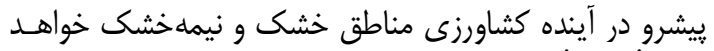

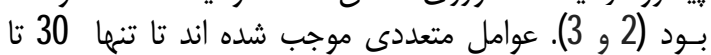

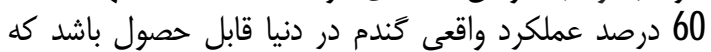

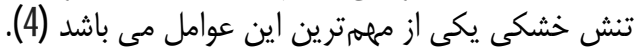

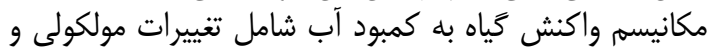

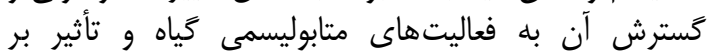

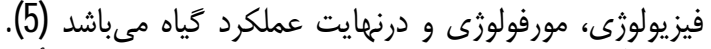

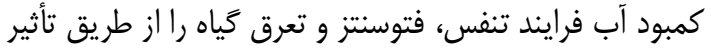
بر آماس سلولى و درنتيجه باز و بسته شدن وند روزنهاه رازها تحت تائر 


\section{نتايج و بحث} تجزيه واريانس و مقايسات ميانكَين

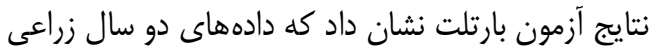

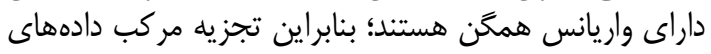

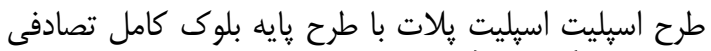
انجام شد (جدول 1). تجزيه واريات بانس بر باسي اساس اميد رياض رياضى تصادى

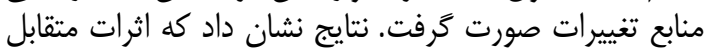

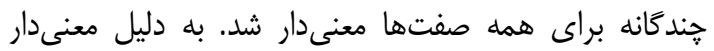

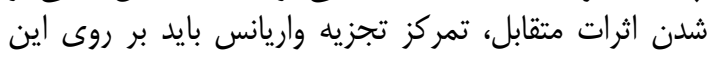

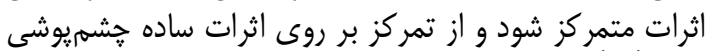

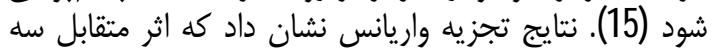

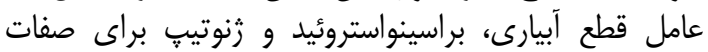

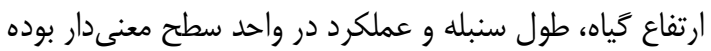

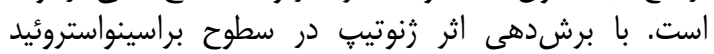

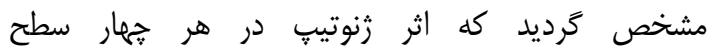

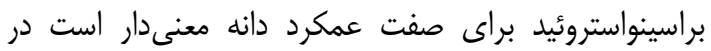

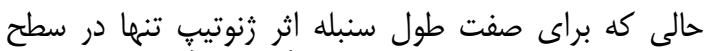

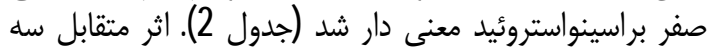

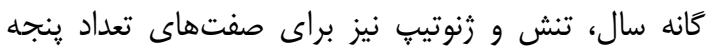

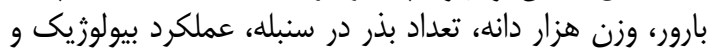

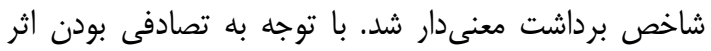

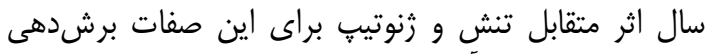

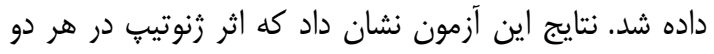

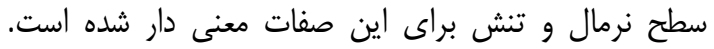

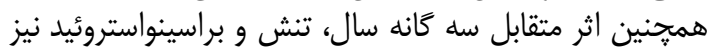

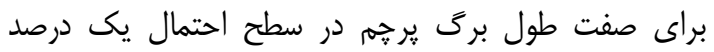

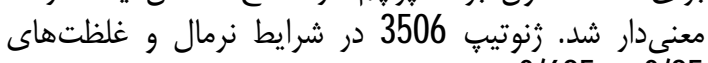

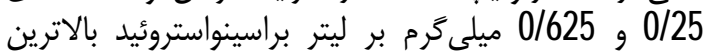

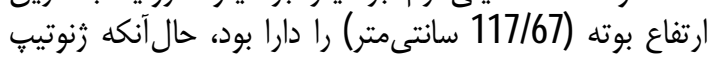

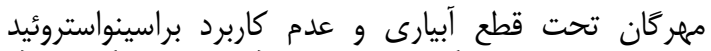

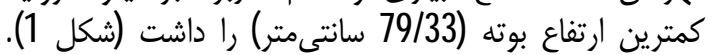

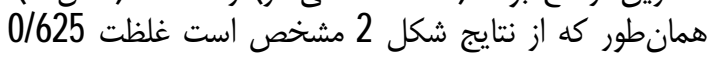

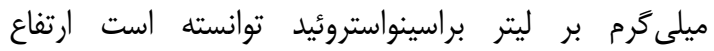
رنوتيِها را تحت تنش افزايش دهد البته اين افزايش ارتفاع

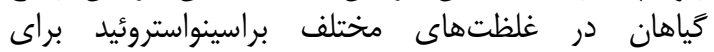

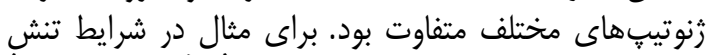

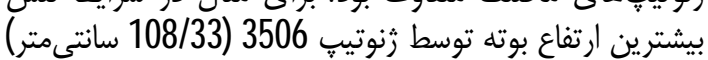

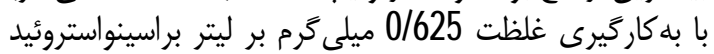

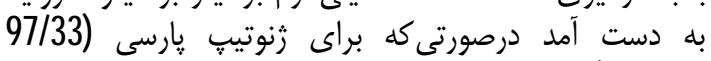

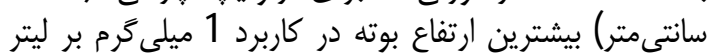

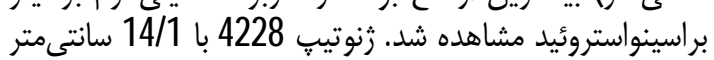

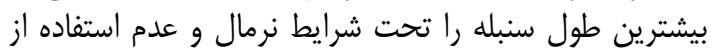

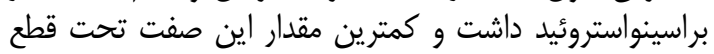

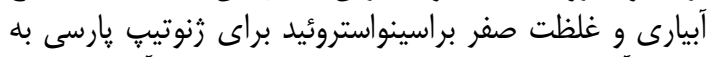

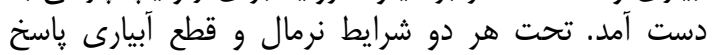

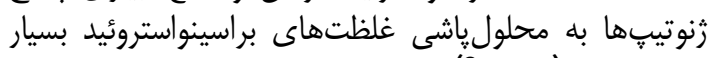

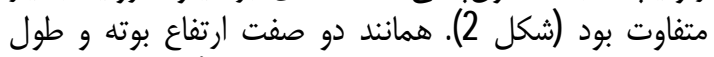

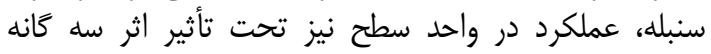
فاكتورهاى موردبررسى قرار كرفت.
جهت بررسى تأثير براسينواستروئيد بر عملكرد و واجزاى

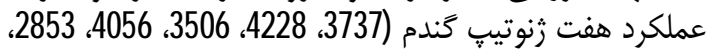

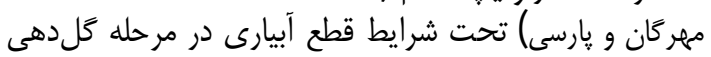

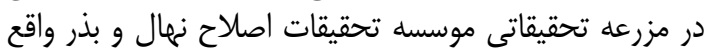

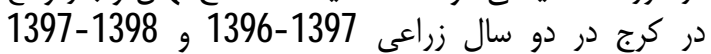

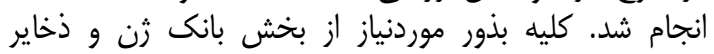

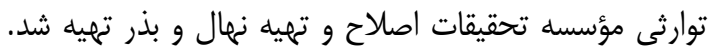

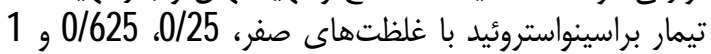

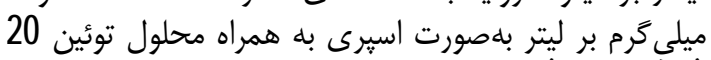
أعمال OO1)

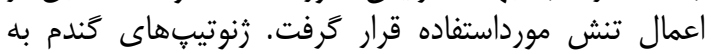

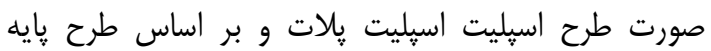

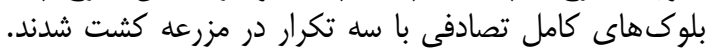

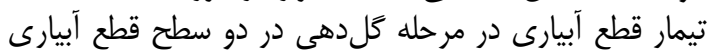

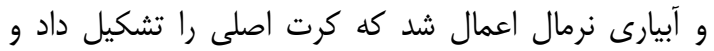

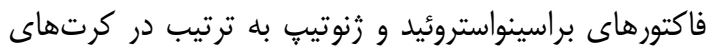
فرعى و فرعى فرعى قرار ترافيند

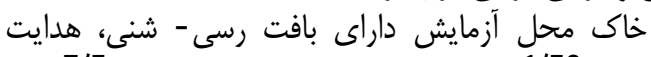

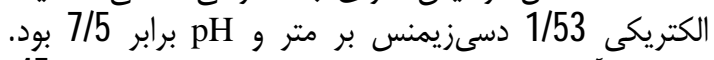

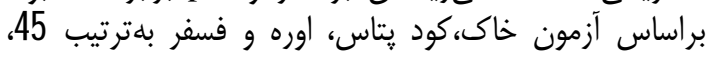
100

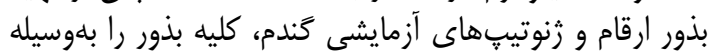

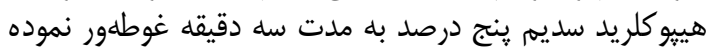

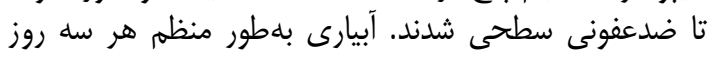

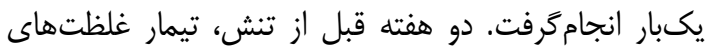

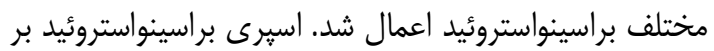

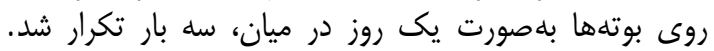

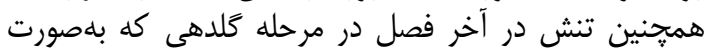

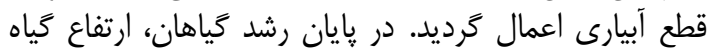

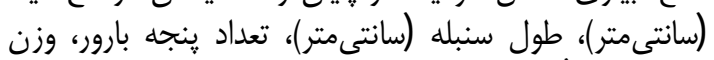

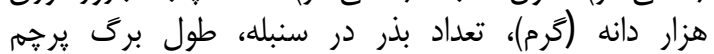

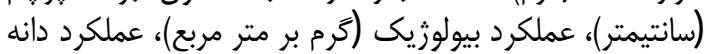

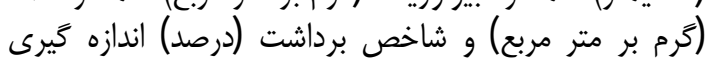

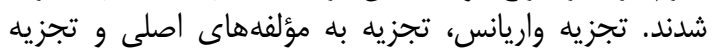

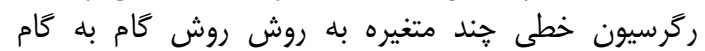
SAS و با استفاده از نرمافزار آمارى روش (Stepwise method)

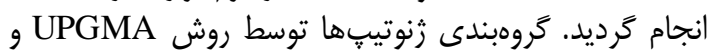

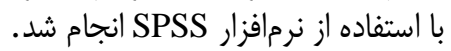

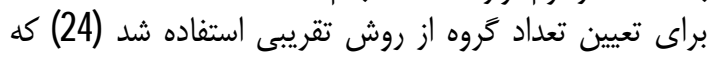

$$
\text { در برابر است با: }
$$

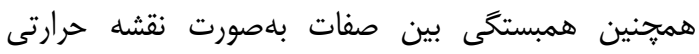

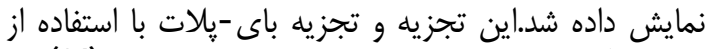

نرمافزار آناين METABOANALYST انجام شدند (14). 
Table 1. ANOVA for measured characters of wheat genotypes

جدول1 - تجزيه واريانس صفات اندازمَيرى شده زنوتيِهاى كَندم

\begin{tabular}{|c|c|c|c|c|c|c|c|c|c|c|}
\hline \multicolumn{9}{|c|}{ ميانكين مربعات Mean square } & \multirow[b]{2}{*}{1} & \multirow{2}{*}{ منابع تغيير S.O.V } \\
\hline$\frac{89}{89}$ & $\frac{Y 8}{C(10=}$ & $\frac{Y 7}{612}$ & $\begin{array}{l}Y 6 \\
7045\end{array}$ & $\begin{array}{l}\mathrm{Y} 5 \\
4 / 88\end{array}$ & $\begin{array}{l}\mathrm{Y4} \\
85 \mathrm{C}\end{array}$ & $\begin{array}{l}\mathrm{Y3} \\
\mathrm{C} / 4 \mathrm{Z}\end{array}$ & $\begin{array}{l}\mathrm{Y} 2 \\
\mathrm{C} / \mathrm{k}\end{array}$ & $\frac{\mathrm{YI}}{14 \mathrm{C}}$ & & \\
\hline 3$] / 2$ & C1004 & C/14 & $17 / 31$ & $14 / 17$ & 4 & C/2 & $2 / 96$ & $\begin{array}{l}145 / 26 \\
16 / 2\end{array}$ & 4 & $\begin{array}{l}Y \\
R(Y)\end{array}$ \\
\hline$\triangle \square O E$ & $3 c / 4$ & $27 / 7$ & $243: / \varepsilon$ & उडAE & $13 / \pi$ & X & 18 & 5AE & ] & A \\
\hline 247 & C/12 & ¿/6 & $171 / 4$ & $8 / 0$ & $79 \pi$ & $Z \mathbb{E} / \mathbb{E}$ & $56 / 35$ & 33" & ] & $\mathrm{Y}^{* \mathrm{~A}}$ \\
\hline $50 / 6 \varepsilon$ & $\mathrm{c} / 00 \mathrm{r}$ & $C / O E$ & $2 / 15$ & $1 / / 017$ & $1 / 19$ & $c / 0 c$ & C/E & $31 / 24$ & 4 & $A^{*} R(Y)$ \\
\hline $116 / \mathrm{C}$ & $C / \equiv$ & Z/WE & $26=17$ & $18 / 31$ & $19 / / 4$ & (/3) & $1 E / 4 k$ & 1325 & $\Xi$ & $\mathrm{B}$ \\
\hline $190 / 2$ & C/014 & $1 / 14$ & $39 / 97$ & $15 / 2 \varepsilon$ & $56 / 1=$ & (/8: & $\Xi / 2 \pi$ & $14 E / 4]$ & $\equiv$ & $\mathrm{Y} * \mathrm{~B}$ \\
\hline $15 E$ & $\mathrm{C} / \mathrm{CL}$ & $\mathrm{C} / \mathrm{C}$ & $14 / 6$ & $145 / T$ & $58 / 6$ & $2 / 0 E$ & $C / 3 E$ & $107 / 2$ & $\Xi$ & $\mathrm{Y}^{*} \mathrm{~A} * \mathrm{~B}$ \\
\hline $28 \mathrm{E} / \mathrm{C}$ & C/EE & $2 / \Xi$ & $30 / 21$ & $230 / 5=$ & $306 / 3$ & $4 / 54$ & $\Sigma / G$ & $107 / \&$ & $\Xi$ & $\mathrm{A} * \mathrm{~B}$ \\
\hline $16 / g_{2}$ & $\mathrm{C} / \mathrm{OI}$ & C/15 & $\mathrm{c} / \overline{1}$ & $16 / 21$ & $1 / 54$ & C/4 & $2 / \pi$ & $150 / 17$ & 24 & $\mathrm{~A} * \mathrm{~B} * \mathrm{R}(\mathrm{Y})$ \\
\hline $311 / 4 k$ & C/9 & $\equiv / \varepsilon$ & $11 / 51$ & $13=/ \pi$ & $3 \% / 5$ & $2 / 28$ & $\varepsilon / \pi$ & 1706 & $\epsilon$ & $\mathrm{G}$ \\
\hline $14=/ 5 E$ & $(/ 12$ & $1 / 0 \mathrm{~A}$ & $24 / 6$ & $6 / 6=$ & $128 / 5$ & $\Xi / 乙$ & $\Xi / 14$ & $42 / 24$ & $\epsilon$ & $\mathrm{Y}^{* \mathrm{G}}$ \\
\hline 216 & $\mathrm{c} / 02$ & $1 / 15$ & $11 / 04$ & $4 E / 2=$ & $18 / \equiv$ & $2 / 2$ & $5 / \pi$ & 10E/TE & $\epsilon$ & $\mathrm{Y} * \mathrm{G} * \mathrm{~A}$ \\
\hline $56 / 42$ & $C / 26$ & $1 / 9 E$ & $7 / 62$ & $171 / 5]$ & 13//6 & $1 / 27$ & ع/01 & $504 / 0=$ & $\epsilon$ & $\mathrm{G}^{*} \mathrm{~A}$ \\
\hline 5$] / 9$ & C/O15 & $C / 2$ & E/6] & 31/11 & $55 / \&$ & C/4E & $2 / 8=$ & $14 / / \mathrm{C}^{-}$ & $1 \varepsilon$ & $\mathrm{Y} * \mathrm{G} * \mathrm{~B}$ \\
\hline$\varpi / \mathscr{L}$ & $\mathrm{c} / \mathrm{0}]$ & $c / 21$ & $2 \pi / 32$ & $46 / 9=$ & $\varpi / 15$ & C/ש & C/\&6 & $23 / \mathrm{G}$ & $1 \varepsilon$ & $\mathrm{G}^{*} \mathrm{~B}$ \\
\hline $3^{5} / X^{2}$ & C/14 & C/IE & $34 / 2$ & $\mathbb{1} / \mathbb{C}$ & $๘ / \notin$ & c/6/ & $11 / 6$ & 19 & $1 \varepsilon$ & $\mathrm{G}^{*} \mathrm{~A} * \mathrm{~B}$ \\
\hline$\pi / 5$ & $C / 0 z$ & $C / 3=$ & $2 \mathrm{C} / \mathrm{]}$ & $1 \varepsilon / \Xi$ & $6 \in / \subseteq$ & $C / 42$ & $\exists / 8$ & $\sigma_{2} / 9_{2}$ & $1 \varepsilon$ & $\mathrm{Y}^{*} \mathrm{G}^{*} \mathrm{~A} * \mathrm{~B}$ \\
\hline $14 / \varepsilon$ & C/OOE & C/OC & $\varepsilon / E$ & $1 / / 4 \varepsilon$ & $I=/ 94$ & C/32 & ]/E & $7 \mathrm{7} / 9]$ & 192 & Error \\
\hline $1=8=$ & ह/42 & $7 / 8$ & $14 / 45$ & घट & $1 / 71$ & $1 / \mathrm{C}$ & $I / 82$ & $\varepsilon ; 3$ & - & $\mathrm{CV}(\%)$ \\
\hline
\end{tabular}

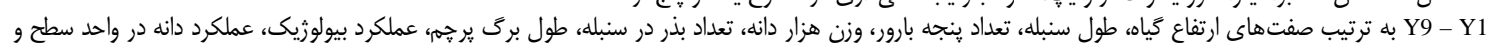

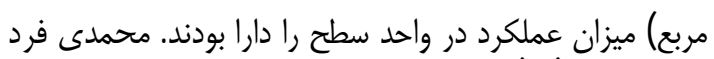

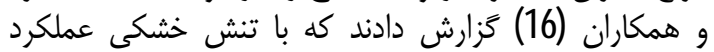

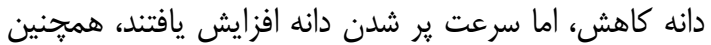

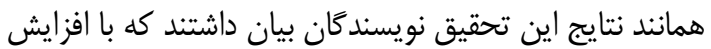

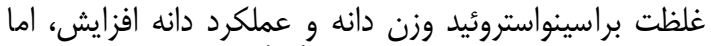

سرعت ير شدن دانه كاهش يافتند (16).

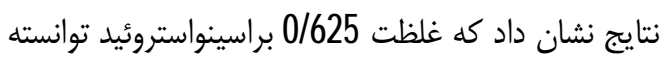

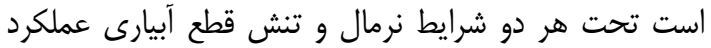

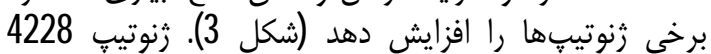

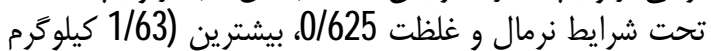

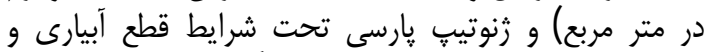

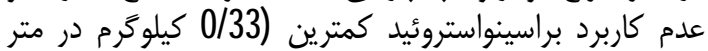

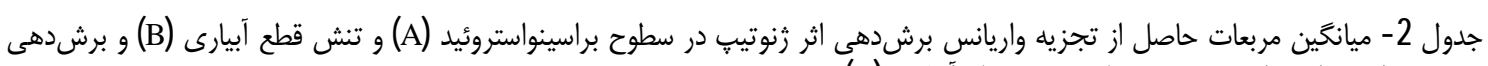

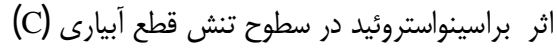

Table 2. Mean squares of slicing interactions between genotype in brassinosteroid levels (A) and water deficit levels

\begin{tabular}{|c|c|c|c|c|c|c|c|c|c|c|c|c|}
\hline Y9 & Y8 & Y7 & Y6 & Y5 & Y4 & Y3 & $\mathrm{Y} 2$ & Y1 & df & S.O.V & سطح & تجزيه \\
\hline- & $\mathrm{C} / \mathrm{CT}$ & - & - & - & - & - & $2 / \pi$ & $255 / 4$ & $E$ & ثنوتب & $C$ & \multirow{4}{*}{ A } \\
\hline - & $C / 06 E^{*}$ & - & - & - & - & - & $7 / 0 C$ & $24=/ 4$ & $\epsilon$ & رنوتيتي & $C / Z$ & \\
\hline- & $\mathrm{C} / \mathrm{OE}^{*}$ & - & - & - & - & - & $16 / 15$ & $94 / 01$ & $\epsilon$ & رَنوتينِ & C/6E & \\
\hline- & $\mathrm{C} / \mathrm{OA}^{*}$ & - & - & - & - & - & $2 / 01$ & 21 & $\epsilon$ & رنوتيبِ & 1 & \\
\hline $18 / 6$ & - & 06 & - & $13 / 88$ & $17 / 7$ & 03 & - & - & 6 & رنوتيب & أبيارى & \multirow{2}{*}{ B } \\
\hline $27 / 36$ & - & $\alpha 1^{*}$ & - & 凹/78 & $41 / 8$ & 013 & - & - & 6 & رنوتيب & قطيارى & \\
\hline- & - & $C / E$ & $14^{*}$ & - & - & - & C/8E & - & $\Xi$ & براسينواستروئيد & نرمال & \multirow[b]{2}{*}{$\mathrm{C}$} \\
\hline - & - & Q1006 & $7 / 57^{*}$ & - & - & - & $7 / 6$ & - & 3 & براسينواستروئيد & قآبيارى & \\
\hline
\end{tabular}

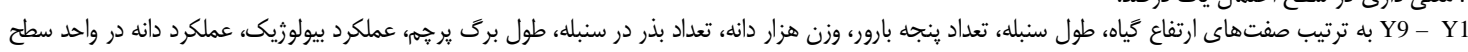




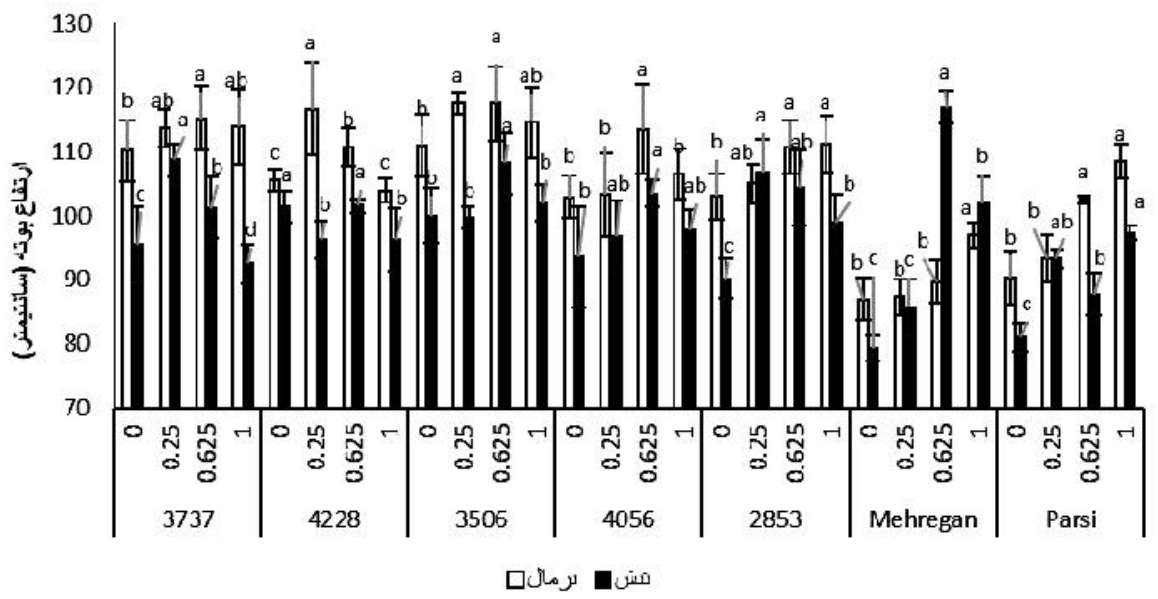

شكل ا- تأثير اثر متقابل براسينواستروئيد و زنوتيب تحت شرايط نرمال و تنش بر روى ارتفاع بوته

Figure 1. Interaction effects of brassinosteroid and genotype on plant height under normal and stress conditions

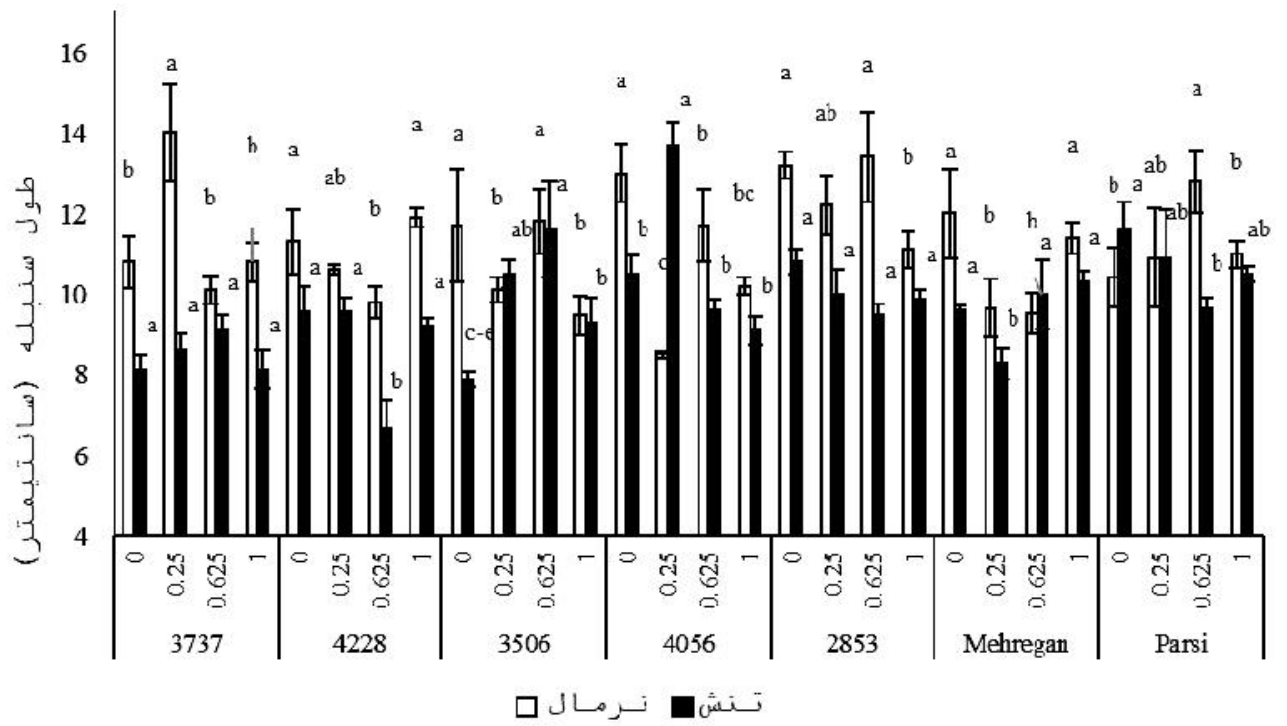

شكل r- تأثير اثر متقابل براسينواستروئيد و زنوتيت تحت شرايط نرمال و تنش بر روى طول سنبله

Figure 2. Interaction effects of brassinosteroid and genotype on spike length under normal and stress conditions 


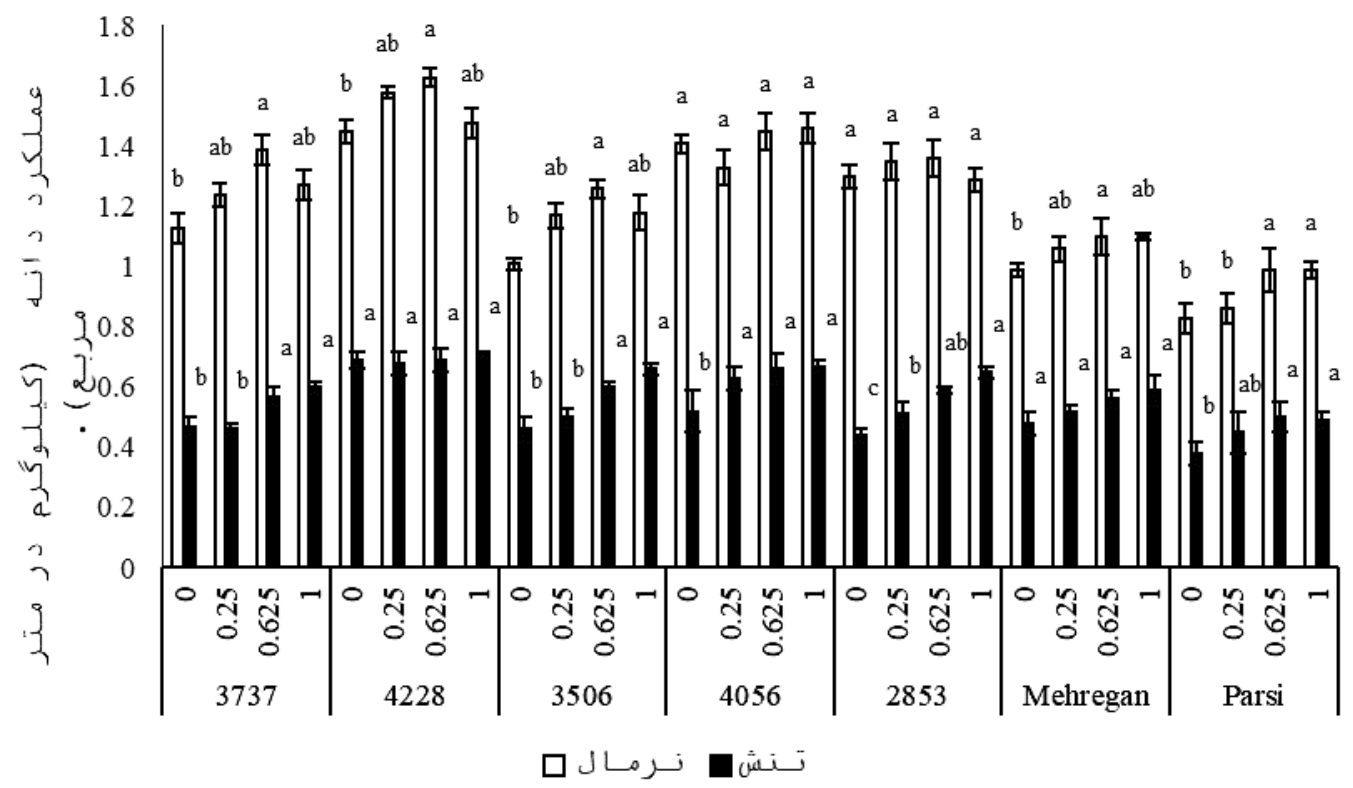

شكل3 - تأثير اثر متقابل براسينواستروئيد و زنوتيب تحت شرايط نرمال و تنش بر روى عملكرد دانه

Figure 3. Interaction effects of brassinosteroid and genotype on grain yield under normal and stress conditions

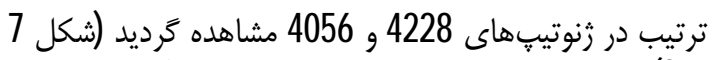
و 8). نتايج اين تحقيق نشان داد كه قطع آبيارى موجب

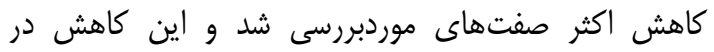

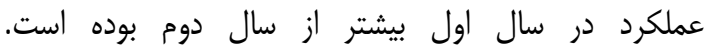

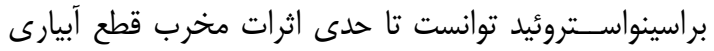

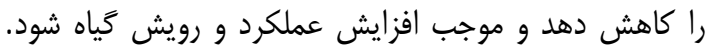
قطع آبيارى به طور معنى دارى عملكرد دانه، وزئه وزن هزار دار دانه،

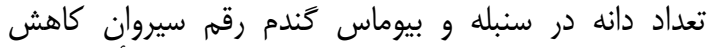
دادهاست، هرجند محلولئي

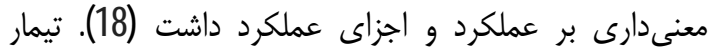

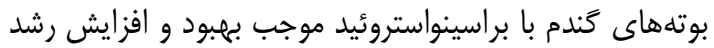

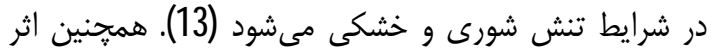

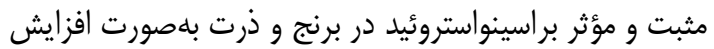

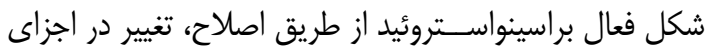

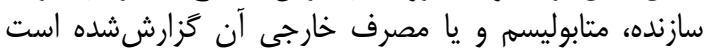

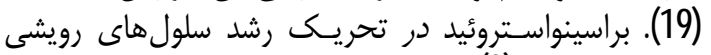

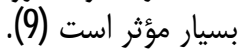

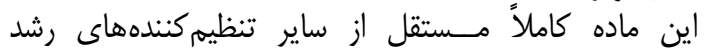

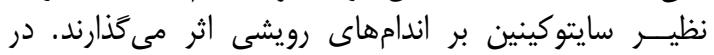

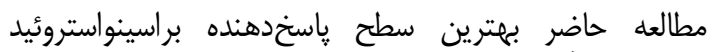

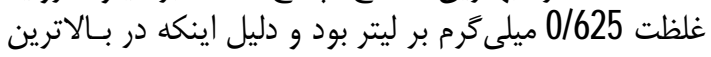

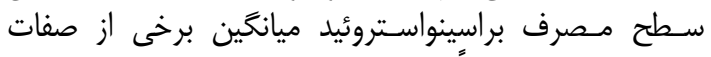

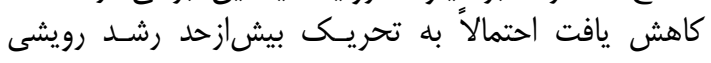

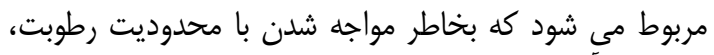
اثر مثبت آن كاهش يافته است.
كياهان درمجموع در سال دوم عملكرد و رويش بهترى

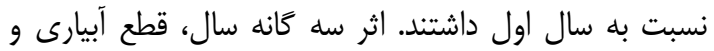

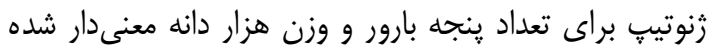

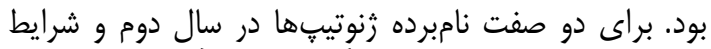

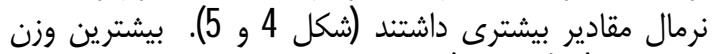

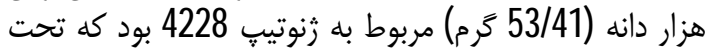

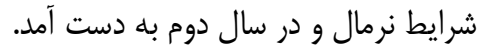
هر جند در اين تحقيق براسينواستروئيد اثرى بر وزن هزار دار دانه

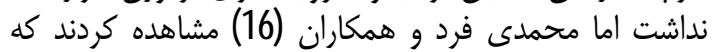

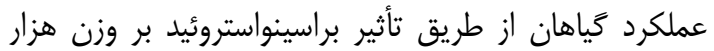

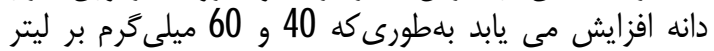

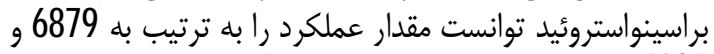

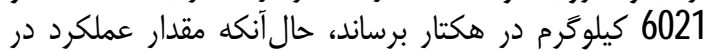

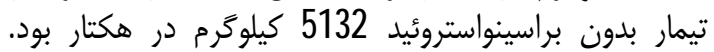

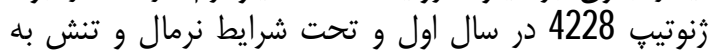

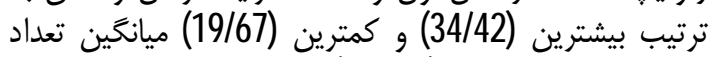

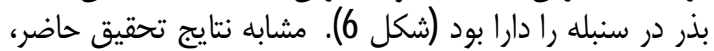

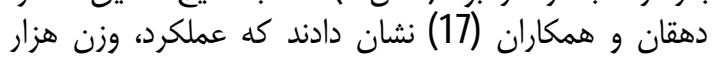

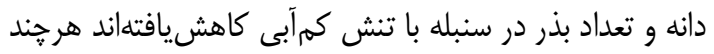

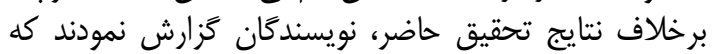

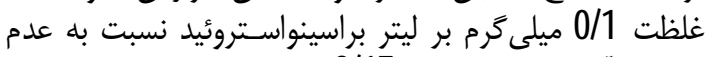

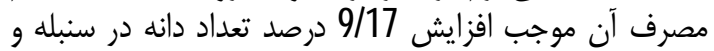

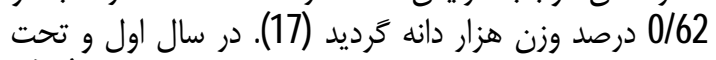

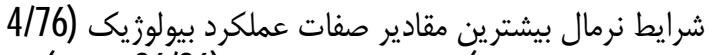
كيلوكَرم بر مترمربح) و شاخص برداشت (36/26 درصد) به 


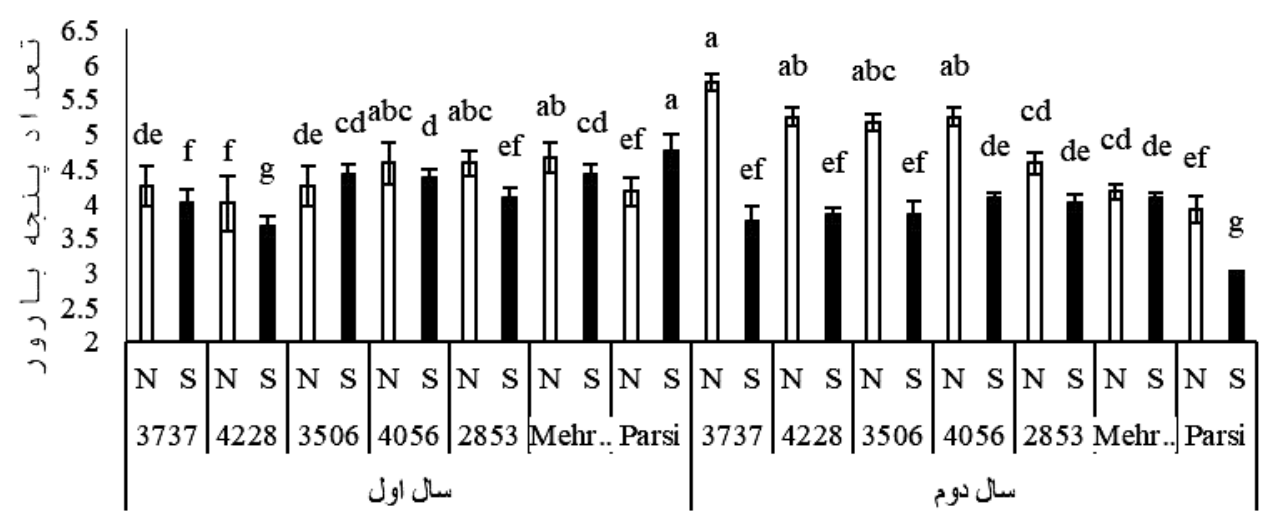

شكل4 - تأثير اثر متقابل تنش قطع آبيارى (N نرمال و S: تنش) و رنوتيب طى دو سال بر روى تعداد بنجهه بارور

Figure 4. Interaction effects of water deficit and genotype on fertilized tiller number during the two years

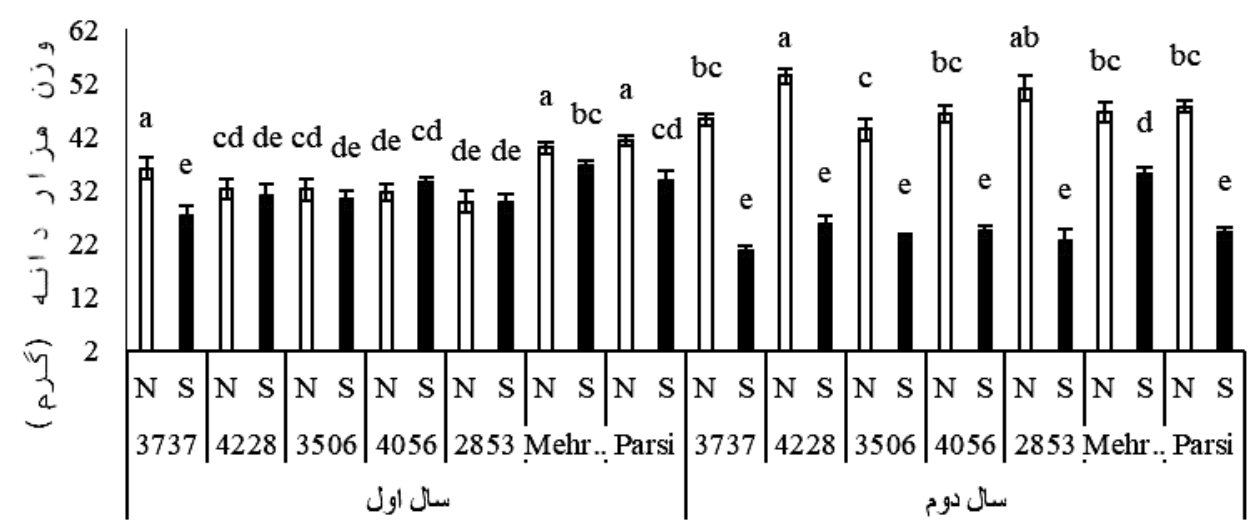

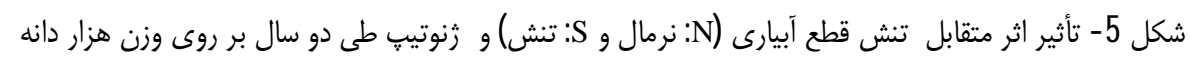

Figure 5. Interaction effects of water deficit and genotype on 1000-seeds weight during the two years

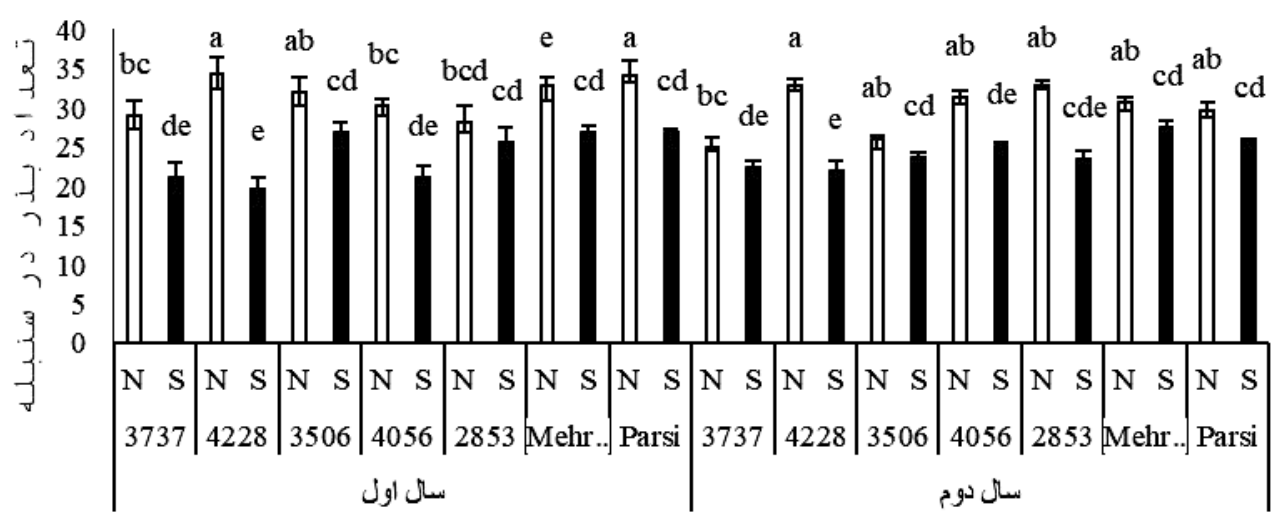

شكل6 - تأثير اثر متقابل تنش قطع آبيارى (N) نرمال و S تنش) و زنوتيب طى دو سال بر روى تعداد بذر در سنبله

Figure 6. Interaction effects of water deficit and genotype on grain number per spike during the two years 


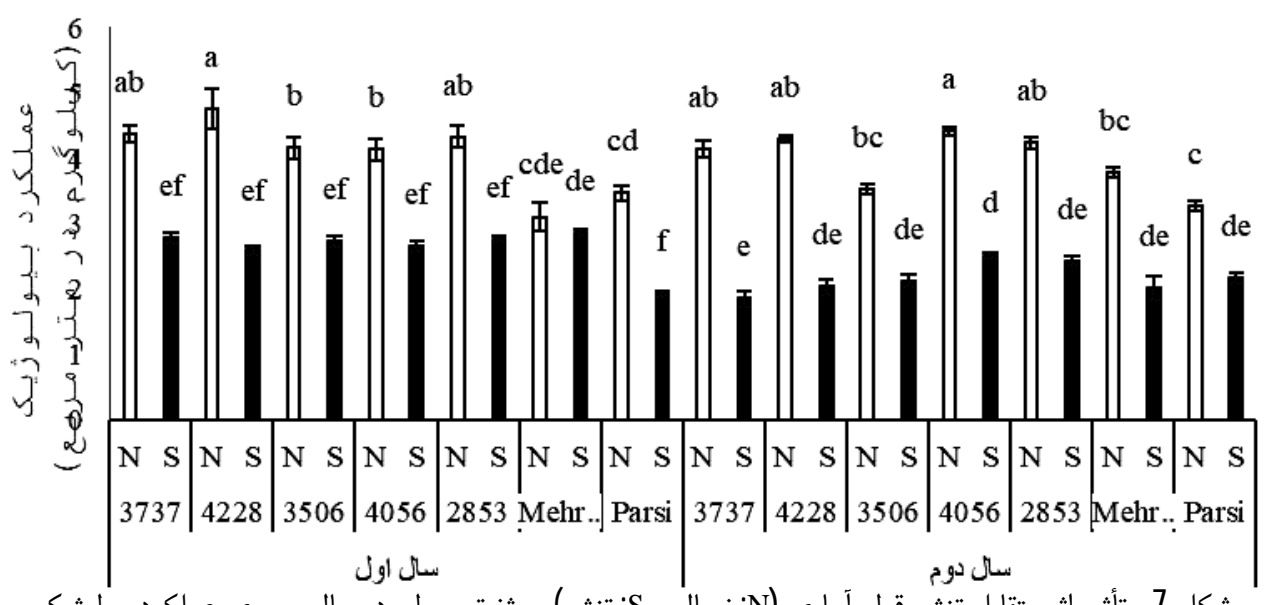

شكل 7 - تأثير اثر متقابل تنش قطع آبيارى (N) نرمال و S تنش) و رنوتيب طى دو سال بر روى عملكرد بيولوزيك

Figure 7. Interaction effects of water deficit and genotype on biological yield $\left(\mathrm{kg} \mathrm{m}^{-2}\right)$ during the two years

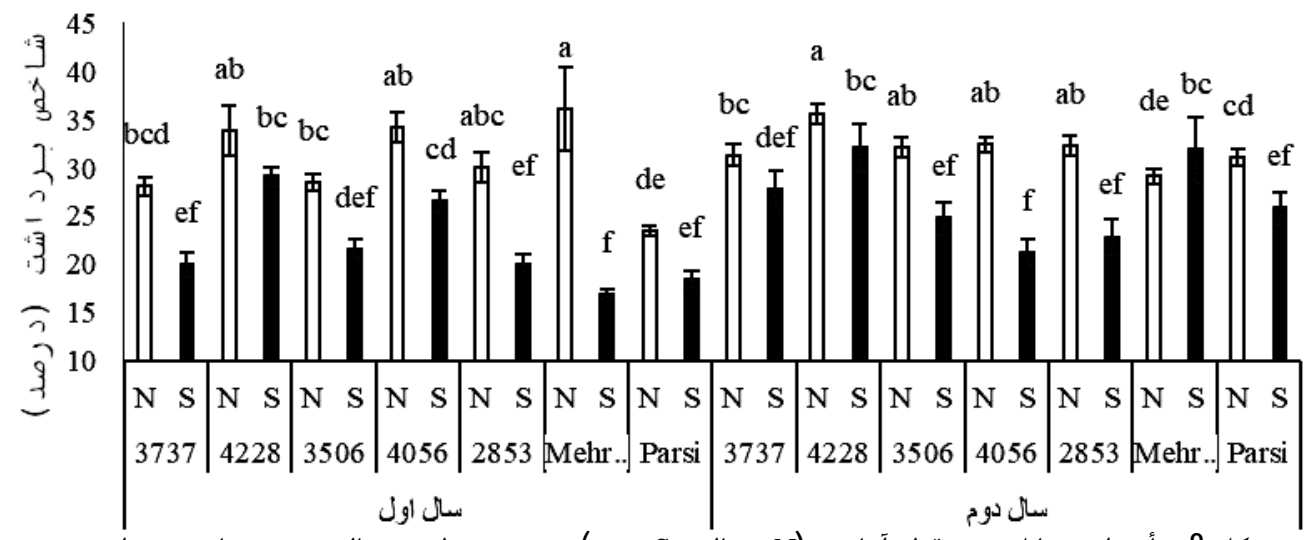

شكل8 - تأثير اثر متقابل تنش قطع آبيارى N ن نرمال و S تنش) و زنوتيب طى دو سال بر روى شاخص برداشت

Figure 8. Interaction effects of water deficit and genotype on harvest index during the two years

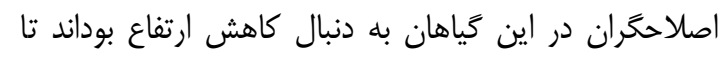

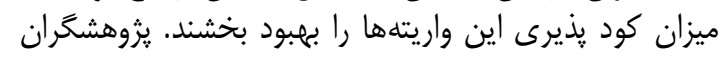

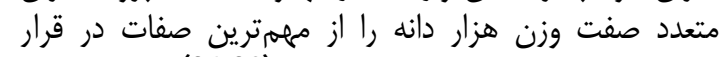

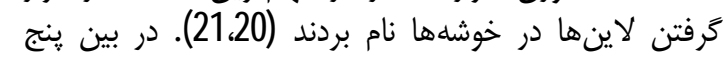

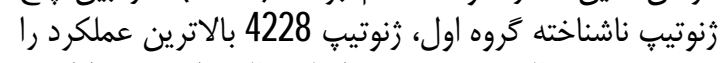

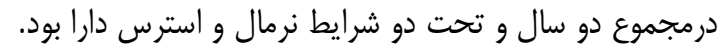

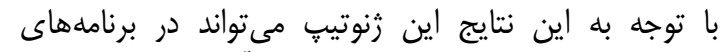

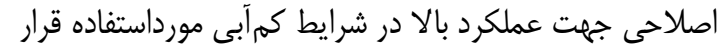

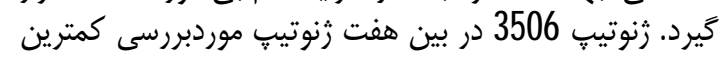

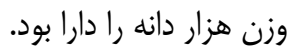

تجزيه خوشهاى و تجزيه به عاملها

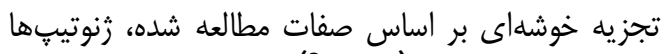

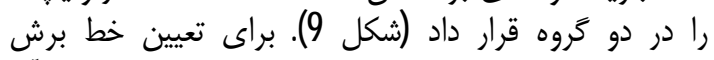

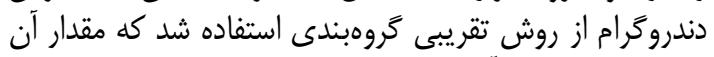

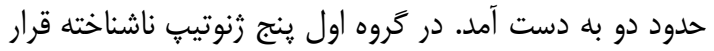

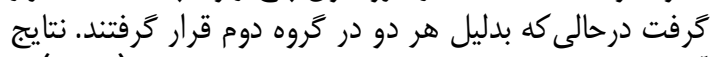

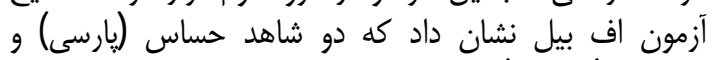

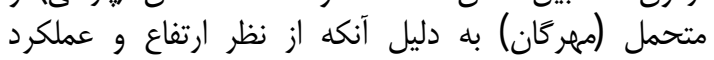

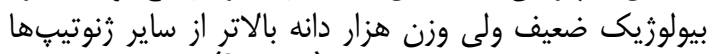

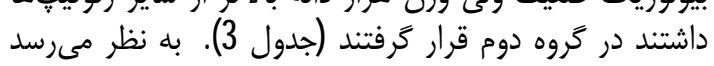




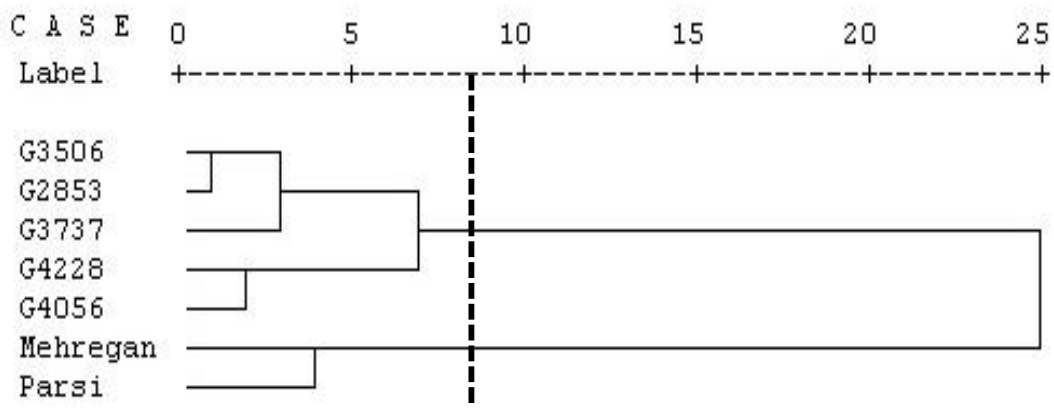

شPGMA شل 9 - دندروخرام صفات موردمطالعه با استفاده از روش UPG

Figure 9. The results of cluster analysis based on measured traits using UPGMA method

اصلى سوم بيش از10 درصد از تغييرات كل را توجيه كرد اين

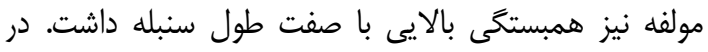

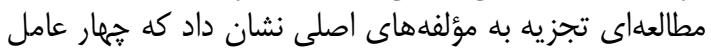

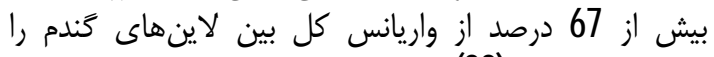

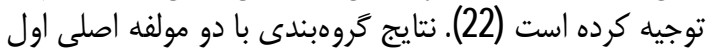

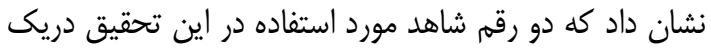

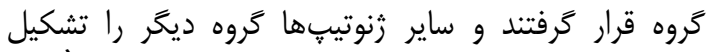

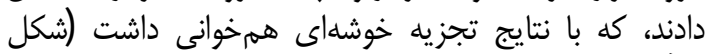

از روش تجزيه به مولفههاى اصلى براى درى روابط

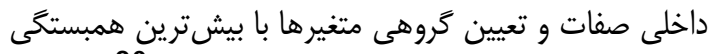

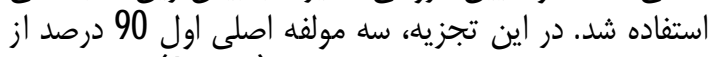

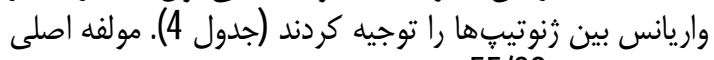

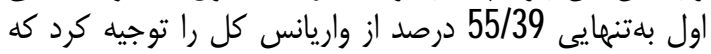

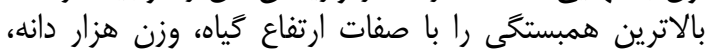

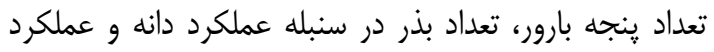

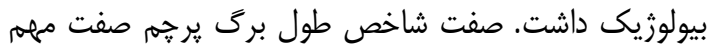

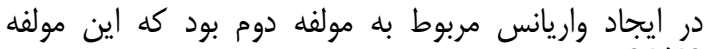

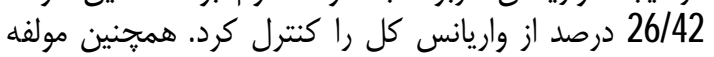

جدول3 - نتايج آزمون اف بيل براى مقايسه دو كروه بدستآمده از تجزيه كلاستر Table 3. The results of F-Beale test for the two groups derived from cluster analysis

\begin{tabular}{|c|c|c|c|c|c|c|c|c|c|c|}
\hline \multicolumn{9}{|c|}{ Mean square ميانكين مربعات } & \multirow{2}{*}{ df } & \multirow{2}{*}{ منابع تغيير } \\
\hline Y9 & Y8 & Y7 & Y6 & Y5 & $\mathrm{Y} 4$ & Y3 & $\mathrm{Y} 2$ & Y1 & & \\
\hline $3 / 74$ & $C / O C$ & $C / 3 E$ & C/24 & $10 / 17$ & $30 / 54$ & C/14 & $C / O E$ & $18 C / 0 E$ & ] & كروه \\
\hline 7/04 & $\mathrm{C} / \mathrm{O}]$ & $\mathrm{C} / \mathrm{CL}$ & $C / 2$ & $\Xi / \Xi$ & $2 / 34$ & $C / O=$ & C/1E & $5 / 45$ & 5 & خطا \\
\hline
\end{tabular}

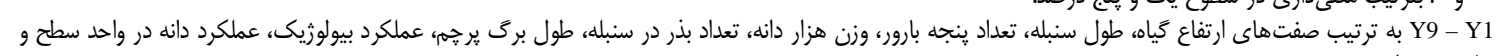

جدول4 - تجزيه به مولفههاى اصلى بر اساس صفات مطالعه شده در اين تحقيق

Table 4. Principal components analysis (PCA) based on studied traits in this study

\begin{tabular}{|c|c|c|c|}
\hline مولفه اصلى سوم & مولفه اصلى دوم & مولفه اصلى اول & صفات \\
\hline C/Ii & $-\mathrm{C} / 2$ & C/92 & ارتفاع گياه \\
\hline$-(/ / 2 \mathcal{E}$ & C/3A & $-C / \mathscr{C}$ & وزن هزار دانه \\
\hline C/14 & C/24 & $-C / \mathscr{C}$ & تعداد بذر در سنبله \\
\hline$-(/ 3]$ & $C / 5 C$ & $C / \pi$ & عملكرد بيولوزيك \\
\hline$C / 1=$ & $C / 66$ & c/6 & تعداد ينجه بارور \\
\hline$-(/ 3]$ & $C / 58$ & $C / \pi$ & عملكُ د دانه \\
\hline $\mathrm{C} / \mathrm{TL}$ & $\mathrm{C} / \mathbf{Q}$ & $-(/ 3 C$ & طول سنبله \\
\hline$C / 2$ & $-\mathrm{C} / 71$ & C/47 & طول بركَ يرجهم \\
\hline 10/8 & 26142 & $55 / 36$ & واريانس نسبى توجيه شدهوها \\
\hline 9/6] & 81/81 & $55 / 39$ & واريانس تجمعى توجيه شدهو\%) \\
\hline
\end{tabular}




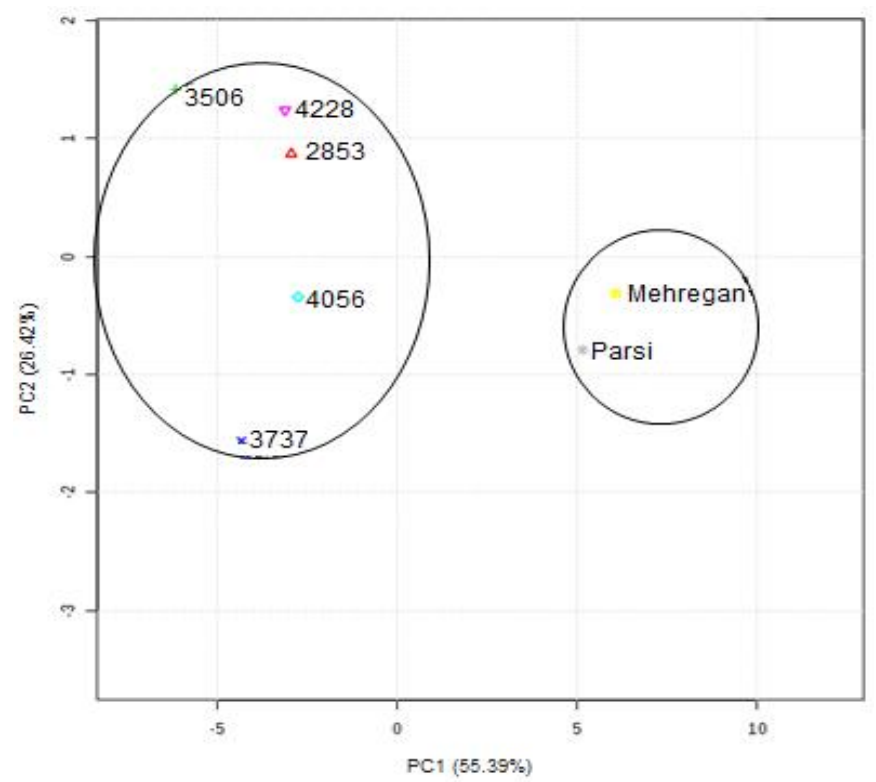

شكل 10 - باى يالات بدست آمده از تجزيه به مولفه هاى اصلى بر اساس تمامى صفات

Figure 10. Biplot derived by PCA procedure based on all measured traits

صفت وزن صد دانه در حالت نرمال بيشترين اهميت را در ارد

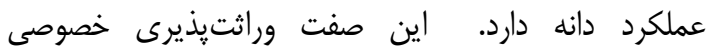

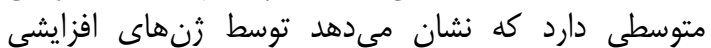

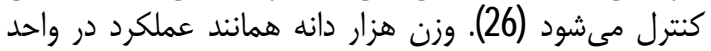

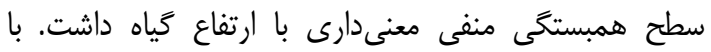

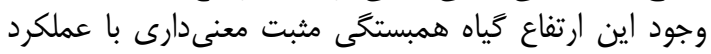

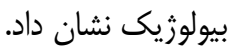
بر اساس نتايج بلهدست آمده از اين تحقيق براسينواستروئيد

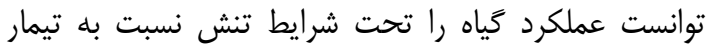

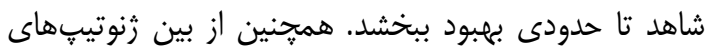

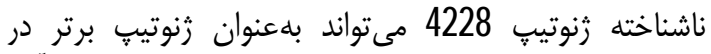

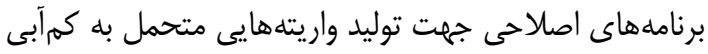

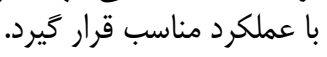

تجزيه رتر سيون و همبستكى بين صفات

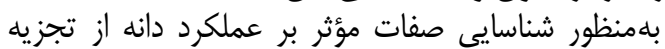

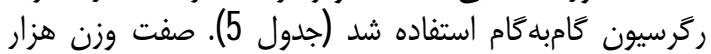

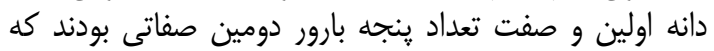

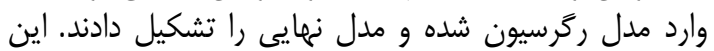

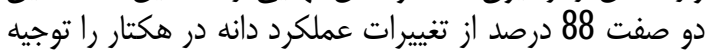

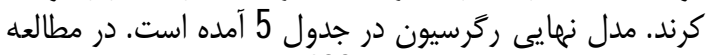

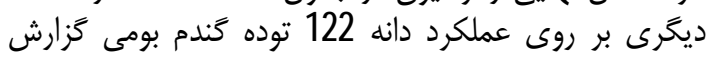

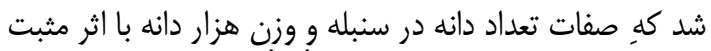

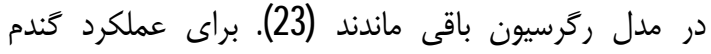

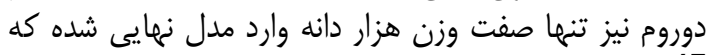

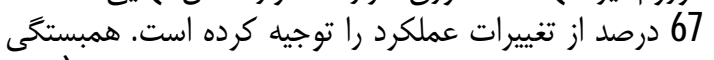

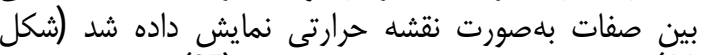
11). تجزيه عليت خسروى و همكاران (25) نشان دادند كهان (شكل

Table 5. The results of step by stepwise regression for yield of wheat genotypes

جدول 5 - نتايج تجزيه رگرسيون گامبهَام براى عملكرد زنوتيبهاى گندم

\begin{tabular}{|c|c|c|c|c|}
\hline ضريب تبيين & ضريب ركرسيون & عرض از مبداً & صفت & مرحله \\
\hline पाI & 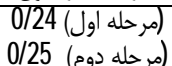 & $-1 / 21$ & وزن هزار دانه (X1) & اول \\
\hline$C / \&$ & $C / x$ & $-\varepsilon / 27$ & تعداد ينجه بارور (X2) & دوم \\
\hline
\end{tabular}




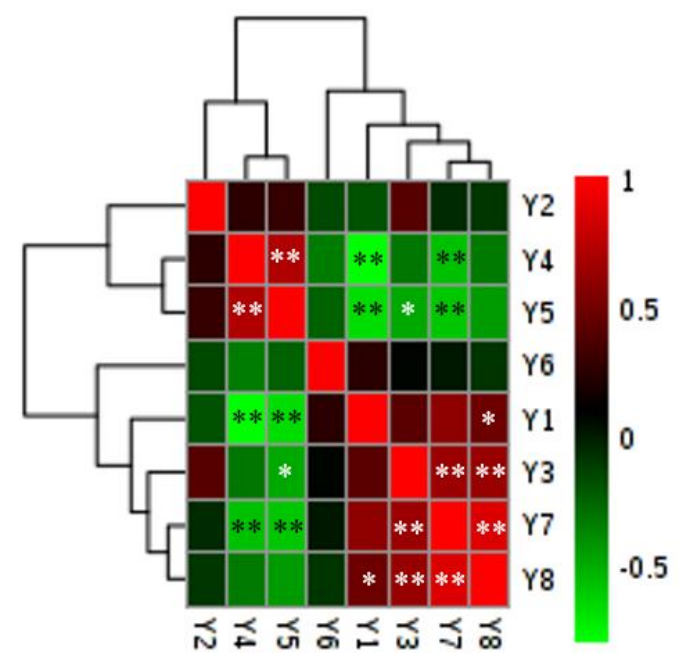

$$
\text { شكل } 11 \text { - نقشه حرارتى همبستخى بين صفات موردمطالعه (" و *:*ترتيب معنى دارى در سطوح هنج و يك درصد) }
$$

Figure 11. Demo-gram of correlation analysis among the studied traits (* and ** indicate significance at 5 and $1 \%$ levels, respectively)

1. Shahbazian, N., A. Dadi and H. Iran Nejad. 2007. Reaction of winter wheat response to previous culture (fallow, wheat, soybeans and alfalfa) and manure application in Qazvin region. Agricultural Sciences, 13(1): 125-135 (In Persian).

2. Sheikh-Mohamadi, M.H., N. Etemadi, A. Nikbakht, M. Faraipour, M. Arab and M.M. Majidi. 2017. Screening and selection of twenty Iranian wheatgrass genotypes for tolerance to salinity stress during seed germination and seedling growth stage. HortScience, 52(8): 1125-1134.

3. Sheikh-Mohamadi, M.H., N. Etemadi, A. Nikbakht, M. Farajpour, M. Arab and M.M. Majidi. 2018. Wheatgrass germination and seedling growth under osmotic stress. Agronomy Journal, 110(2): 572585.

4. Deng, X.P., L. Shan, S.Z. Kang and I. Shinobu. 2003. Improvement of wheat water use efficiency in semiarid area of China. Agriculture Science China, 2: 35-44.

5. Wang, L., Z. Guo, Y. Zhang, Y. Wang, G. Yang, L. Yang, R. Wang and Z. Xie. 2017. Characterization of LhSorP5CS, a gene catalyzing proline synthesis in oriental hybrid lily sorbonne: molecular modelling and expression analysis. Botanical Studies, 58: 1-10.

6. Shekoofa, A. and T. Sinclair. 2018. Aquaporin activity to improve crop drought tolerance. Cells, 7(9): 123.

7. Senapati, N., P. Stratonovitch, M.J. Paul and M.A. Semenov. 2018. Drought tolerance during reproductive development is important for increasing wheat yield potential under climate change in Europe. Journal of Experimental Botany, 70(9): 2549-2560.

8. Mega, R., F. Abe, J.S. Kim, Y. Tsuboi, K. Tanaka, H. Kobayashi and S.R. Cutler. 2019. Tuning wateruse efficiency and drought tolerance in wheat using abscisic acid receptors. Nature plants, 5(2): 153.

9. Peres, A.L.G., J.S. Soares, R.G. Tavares, G. Righetto, M.A. Zullo, N.B. Mandava and M. Menossi. 2019. Brassinosteroids, the sixth class of phytohormones: a molecular view from the discovery to hormonal interactions in plant development and stress adaptation. International Journal of Molecular Sciences, 20(2): 331.

10. Xi, Z., Z. Zhang, S. Huo, L. Luan, X. Gao, L. Ma and Y. Fang. 2013. Regulating the secondary metabolism in grape berry using exogenous 24-epibrassinolide for enhanced phenolics content and antioxidant capacity. Food Chemistry, 141: 3056-3065.

11. Shahid, M., M. Pervez, R. Balal, N. Mattson, A. Rashid, R. Ahmad, C. Ayyub and T. Abbas. 2011. Brassinosteroid (24-Epibrassinolide) enhances growth and alleviates the deleterious effects induced by salt stress in pea (Pisum sativum L.). Australian Journal of Crop Science, 5: 500-510.

12. Hussain, M., T.A. Khan, M. Yusuf and Q. Fariduddin. 2019. Silicon-mediated role of 24epibrassinolide in wheat under high-temperature stress. Environmental Science and Pollution Research, 1-10.

13. Ashraf, M., N. Akram, R. Arteca and M. Foolad. 2010. The physiological, biochemical and molecular roles of brassinosteroids and salicylic acid in plant processes and salt tolerance. Critical Reviews in Plant Sciences, 29: 162-190.

14. Xia, J. and D.S. Wishart. 2016. Using MetaboAnalyst 3.0 for comprehensive metabolomics data analysis current protocols in bioinformatics. Current Protocol Bioinformatics, 55: 14.10.1-14.10.91. 
15. Wickens, T.D. and G. Keppel. 2004. Design and analysis: A researcher's handbook. Pearson PrenticeHall, New Jersey.

16. Mohamadifard, F., B. Jafari Haghighi and H.R. Miri. 2010. Effect of Brassinosteroid application in yield and its components of bread wheat (Triticum aestivum L.) under post flowering drought stress. National Conference on Water Crisis, Arsanjan branch, Islamic Azad University, Arsanjan, Iran. 1-15.

17. Dehghan, M., H.R. Balouchi, A.R. Yadavi and F. Safikhani. 2017. Effect of foliar application of brassinolide on grain yield and yield components of bread wheat (Triticum aestivum L.) cv. Sirvan under terminal drought stress conditions. Iranian Journal of Crop Sciences, 19(1): 40-56 (In Persian).

18. Naghizadeh, M. and R. Kabiri. 2018. Effect of Brassinosteroid Foliar on yield and Yield Components of Wheat (Sirvan cultivar) under drought stress. The first international conference and the third national conference on sustainable resource management Soil and Environment 13 and 14 September, Kerman (In Persian).

19. Vriet, C., E. Russinova and C. Reuzeau. 2012. Boosting crop yields with plant steroids. The Plant Cell, 24(3): 842-857.

20. Aghaei, S., E. Tohidi-Nejad and M. Nasr-Esfahani. 2015. Assessment of yield and other agronomic traits of in durum wheat genotypes at Isfahan. Journal of Applied Crop Breeding, 3(1): 69-77.

21. Devesh, P., P.K. Moitra, R.S. Shukla and S. Pandey. 2019. Genetic diversity and principal component analyses for yield, yield components and quality traits of advanced lines of wheat. Journal of Pharmacognosy and Phytochemistry, 8(3): 4834-4839.

22. Naghdeipour, A., M. Khoda Rahmi, A. Pourshahbazi and M. Esmailzadeh. 2011. Principal components analysis for yield and other characters of wheat. Agronomy and Plant Breeding Journal, 7(1): 84-96.

23. Aghaee Sarbaze, M. and A. Amini. 2011. Genetic variability for agronomy traits in bread wheat genotype collection of Iran. Seed and Plant, 27: 581-599.

24. Mardia, K.V., J.T. Kent and J.M. Bibby. 1979. Multivariate analysis. Academic press. London, UK.

25. Khosravi, S., R. Azizinezhad, A. Baghizadeh and M. Maleki. 2019. Evaluation of tolerance for drought among a number of wild diploid populations, tetraploid and hexaploid cultivars of wheat using morphological and agronomic traits. Journal of Crop Breeding, 11(31): 11-27 (In Persian).

26. Asadi A.A., M. Valiadeh, S.A. Mohammadi and M. Khodarahmi. 2019. Genetic analysis of some physiological and yield traits in wheat by F3 families analysis under normal and late season water deficit conditions. Journal of Crop Breeding, 11(31): 55-64 (In Persian). 


\title{
Effect of Brassinosteroid Application on Yield and Its Components of Wheat Genotypes Under Water Deficit Conditions
}

\author{
Mehrnoush Rafei ${ }^{1}$, Mohammad Reza Amerian ${ }^{2}$, Behzad Sorkhi Lelah Lou ${ }^{3}$, \\ Parviz Heydari ${ }^{4}$ and Hamid-Reza Asghari ${ }^{1}$ \\ 1- Department of Agronomy, Shahrood University of Technology, Shahrood, Iran \\ 2- Department of Agronomy, Shahrood University of Technology, Shahrood, Iran, (Corresponding author: \\ amerianuk@yahoo.co.uk) \\ 3- Seed and Plant Improvement Institute, Cereals Research Department, AREEO, Karaj, Iran \\ 4- Department of Biotechnology, Shahrood University of Technology, Shahrood, Iran \\ Received: October 31, 2019 \\ Accepted: January 7, 2020
}

\begin{abstract}
Drought is the major abiotic factor limiting crop production over the world. The present study aimed to evaluate the effects of brassinosteroid on yield and its components of seven wheat genotypes under water deficit conditions. A split-split plot design based on a randomized complete block design with three replications in the research filed of Seed and Plant Improvement Institute in Karaj at 2017-2018 and 2018-2019 cropping seasons. The main plots were two levels of water deficit (normal irrigation and irrigation cut off from flowering stage), subplots were four concentrations of brassinosteroid $\left(0,0.25,0.625\right.$ and $\left.1 \mathrm{mg} . .^{-1}\right)$ and sub-sub plots were allocated to six wheat genotypes (3737, 4228, 3506, 4056, 2853, Mehrgan and Parsi). The result revealed that irrigation cut off in flowering stage caused reduction in the mean values of the characters. Also, results demonstrated that the brassinosteroid partially offset the adverse impacts of water deficit, which, application of $0.625 \mathrm{mg} \cdot \mathrm{l}^{-1}$ was more effective than the other levels. The highest grain yield $\left(1.63 \mathrm{~kg} \mathrm{~m}^{-2}\right)$ was obtained in 4228 genotype under normal conditions and application of $0.625 \mathrm{mg} .1^{-1}$ of brassinosteroid, whereas the lowest $\left(0.33 \mathrm{~kg} \mathrm{~m}^{-2}\right)$ was observed in Parsi under water deficit and whiteout brassinosteroid. Based on principal components analysis the three first components explained 92 of total variance among the genotypes. According to the results of present study brassinosteroid partially offset the adverse impacts of water deficit and caused increasing in wheat yield. Also, the results suggested that 4228 genotype can be a candidate for future wheat breeding programs to produce water deficit tolerant varieties with proper yield.
\end{abstract}

Keywords: Foliar application, Flowering, Biological yield, Wheat 